\title{
Synthesis of $[1,2,5]$ selenadiazolo[3,4-f]quinolone derivatives by the Gould-Jacobs reaction of 5-amino-2,1,3-benzoselenadiazole
}

\author{
Maroš Bella, Marcel Schultz, and Viktor Milata* \\ Department of Organic Chemistry, Faculty of Chemical and Food Technology, Radlinského 9, \\ SK-812 37, Bratislava, Slovak Republic \\ E-mail: viktor.milata@stuba.sk
}

Dedicated to Prof. Pawel Kafarski in honor of his scientific achievements within his career

\begin{abstract}
The Gould-Jacobs reaction of 5-amino-2,1,3-benzoselenadiazole afforded solely angularly annulated 8-substituted 9-oxo-6,9-dihydro-[1,2,5]selenadiazolo[3,4-f]quinoline derivatives. In the course of thermal cyclization no linear products were observed. Nucleophilic vinylic substitution of activated enolethers with 5-amino-2,1,3-benzoselenadiazole was also studied.
\end{abstract}

Keywords: Quinolones, Gould-Jacobs reaction, nucleophilic vinylic substitution, selenaheterocycles, 2,1,3-benzoselenadiazoles

\section{Introduction}

4-Oxoquinolines (4-quinolones) belong to a class of heterocyclic compounds with a strong antimicrobial activity widely used in modern medicine as broad-spectrum antibiotics. ${ }^{1,2}$ So far, a vast number of 4-quinolone derivatives has been synthesized in order to increase their antibacterial impact or to study their physicochemical properties. Herein, we report the application of the Gould-Jacobs reaction to 5-amino-2,1,3-benzoselenadiazole yielding only angularly annulated 8-substituted [1,2,5]selenadiazolo[3,4-f]quinolones $\mathbf{1}$. The presence of selenium as a photosensitive element in the structure of 4-quinolones could lead to potential biological/photobiological activity ${ }^{3,4}$ as well as to interesting photochemical and spectral characteristics. $^{5,6}$ On the other hand, it is commonly thought that organic compounds containing selenium are very poisonous and foul but toxicity of weakly volatile selenaheterocycles should be low and they are odourless. ${ }^{7}$ Moreover, selenaheterocyclic compounds have been recently intensively studied due to their positive biological impact and their pharmacology along with toxicology have been thoroughly reviewed. ${ }^{7-9}$

Among selenaheterocycles 2,1,3-benzoselenadiazoles have been employed in the synthesis of nitrogen containing heterocycles by a reductive deselenation to form appropriate benzene-1,2- 
diamine derivatives. The latter ones are readily transformed into benzimidazoles or quinoxalines. ${ }^{10}$ Thus $[1,2,5]$ selenadiazolo[3,4-f]quinolones $\mathbf{1}$ become potential starting materials for a further synthesis of nitrogen heterocycles.

\section{Results and Discussion}

In the synthesis of 4-quinolones is usually employed Gould-Jacobs reaction. ${ }^{11}$ This reaction actually represents a sequence of two reactions. In the first step, an aromatic amine reacts with activated enolether in nucleophilic vynilic substitution ${ }^{12}$ yielding an appropriate enamine which after treatment of heat $\left(260{ }^{\circ} \mathrm{C}\right)$ furnishes a desired 4-quinolone derivative. Thus, the key intermediate in our synthesis towards [1,2,5]selenadiazolo[3,4-f]quinolones 1 represented 5amino-2,1,3-benzoselenadiazole (4). The preparation of amine $\mathbf{4}$ described in the literature employs cyclization of 1,2,4-triaminobenzene dihydrochloride (3) with an appropriate selenium reagent. ${ }^{13,14}$ Reduction of 2,4-dinitroaniline (2) on Raney nickel catalyst using hydrazine hydrate as a source of hydrogen led smoothly to 1,2,4-triaminobenzene. However, free triaminobenzene was not isolated due to its high sensitivity to air, but was immediately transformed into its dihydrochloride 3 by treatment with concentrated hydrochloric acid (Scheme 1). ${ }^{15}$

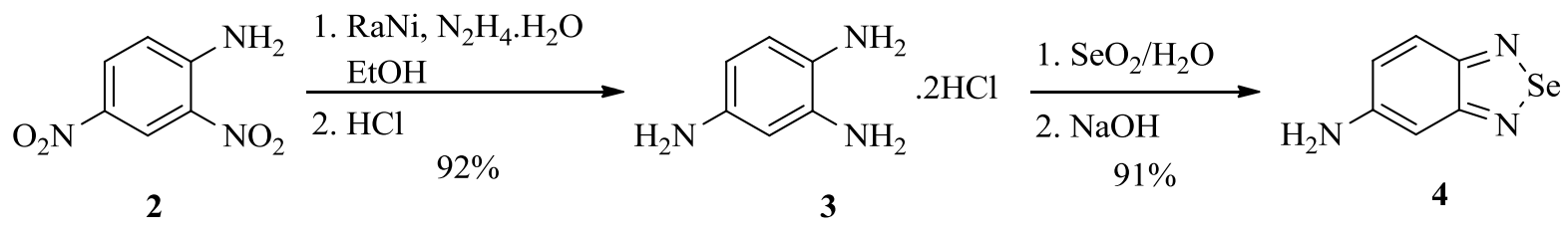

\section{Scheme 1}

Cyclization of dihydrochloride $\mathbf{3}$ with one equivalent of selenium dioxide in water (selenous acid) followed by alkalization of the reaction mixture with sodium hydroxide solution gave amine 4 in $91 \%$ yield.

The obtained amine 4 was used into nucleophilic vinylic substitution with the appropriate activated enolethers 5a-i. The nucleophilic vinylic substitution was carried out in refluxing methanol or ethanol with $10 \%$ excess of activated enolethers $\mathbf{5 a - i}$ and provided a series (benzoselenadiazol-5-ylamino)ethenes 6 in good yields within relatively short reaction times (1030 min.) (Scheme 2). However, cyanoacetic acid derivatives $\mathbf{6 f}, \mathbf{h}$ required much longer reaction times $(16 \mathrm{~h})$ probably due to lower reactivity of the corresponding activated enolethers $\mathbf{5 f}, \mathbf{h}$. Moreover, 2-(ethoxymethylene)malononitrile did not react with amine $\mathbf{4}$ even if the reaction was conducted in refluxing toluene or dimethylformamide. The fact that 2-(ethoxymethylene) malononitrile does not undergo the substitution with amine 4 was verified by quantum-chemical calculations. Standard geometry optimizations of compounds under study at DFT level of theory using hybrid B3LYP functional ${ }^{16}$ and cc-pVDZ basis sets ${ }^{17,18}$ have been performed using 
Gaussian03 program package. ${ }^{19}$ Selected electronic structure data of 2-(ethoxymethylene) malononitrile are summarized in our previous paper. ${ }^{20}$ In this article, we report selected electronic structure data of amine 4 calculated according to the identical methods used for 2(ethoxymethylene)malononitrile (Table 1). Likewise in our previous case, the poor reactivity can be explained by the relatively large energy difference (ca. $3.7 \mathrm{eV}$ ) between HOMO of 4 nucleophile and LUMO of electrophile (2-(ethoxymethylene)malononitrile).

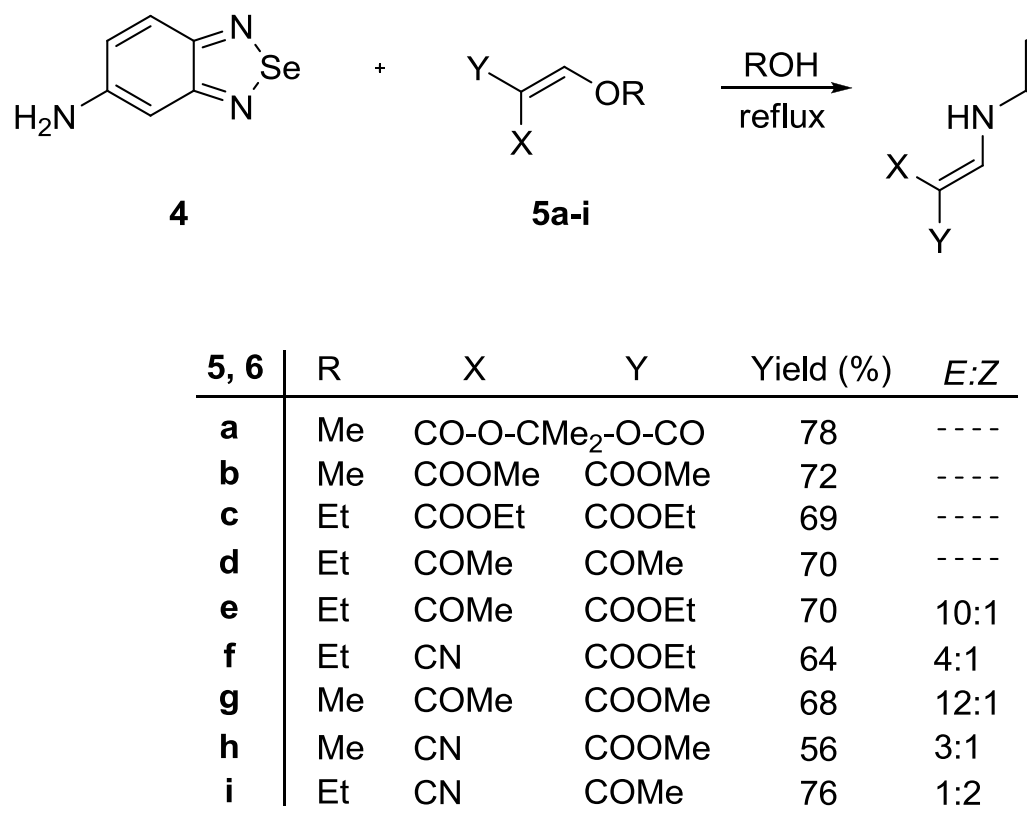

\section{Scheme 2}

Table 1. Selected electronic structure data of 5-amino-2,1,3-benzoselenadiazole (4)

\begin{tabular}{cccc}
\hline FED of atom & Atomic charge & HOMO & LUMO \\
\hline $\mathrm{C}(7)$ & +0.074 & 0.080 & 0.205 \\
$\mathrm{C}(6)$ & +0.062 & 0.005 & 0.209 \\
$\mathrm{C}(4)$ & +0.054 & 0.354 & 0.100 \\
$\mathrm{~N}($ amine $)$ & -0.099 & 0.357 & 0.053 \\
Orbital energy $[\mathrm{eV}]$ & ------ & -5.694 & -2.181 \\
\hline
\end{tabular}

The structure of (benzoselenadiazol-5-ylamino)ethenes 6 was confirmed by means of ${ }^{1} \mathrm{H}$ and ${ }^{13} \mathrm{C}$ NMR spectroscopy. On the basis of high values of vicinal coupling constants ${ }^{3} J_{\mathrm{NH}-\mathrm{CH}}(12-14$ $\mathrm{Hz}$ ) in ${ }^{1} \mathrm{H} \mathrm{NMR}$, an antiperiplanar conformation of $\mathrm{NH}-\mathrm{CH}$ moiety could be assigned. Compounds 6e-i bearing different substituents were isolated as a mixture of $E$ and $Z$ isomers. The relative ratio of the individual geometric isomers was estimated from their ${ }^{1} \mathrm{H}$ NMR spectral data considering intensities of signals. In fact, it is impossible to separate this type of geometrical 
isomers because isomerization occurs in solution, especially if the solvent used is basic. ${ }^{21}$ Although, in all cases both isomers were formed, the $E$ isomers were strongly preferred for enamines $6 \mathbf{e}$ and $\mathbf{6 g}$. The predomination of $E$ isomers is based on the steric repulsions as well as on formation of strong hydrogen bond between amino hydrogen and oxygen of the acetyl group. $^{22}$ The cyanoacetic acid derivatives $\mathbf{6 f}, \mathbf{h}$ also gave mainly $E$ isomers, however this preference is controlled by the steric factors only. In this case, the steric repulsions overcome possible H-bond between amino hydrogen and ester group which seems to be much weaker. ${ }^{22}$ These conclusions are supported by the fact that 3-oxobutanenitrile $\mathbf{6 i}$ afforded preferentially $Z$ isomer. The structure of the compounds $\mathbf{6 a - h}$ was verified by means of EI mass spectroscopy and their fragmentation was investigated. ${ }^{23}$

Selected (benzoselenadiazol-5-ylamino)ethenes 6a-c,e,f containing at least one ester group or its synthetic equivalent (Meldrum's acid derivative) were subjected to thermal cyclization. In principal, the thermal cyclization can provide angularly and/or linearly annulated selenadiazoloquinolones. Despite the possibility of formation of both products, solely angularly annulated selenadiazolo[3,4-f]quinolones 1 were formed and isolated in good yields (Scheme 3).

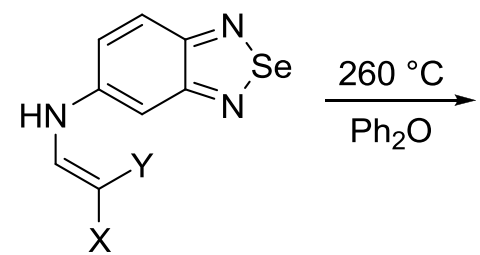

6a-c, e, f

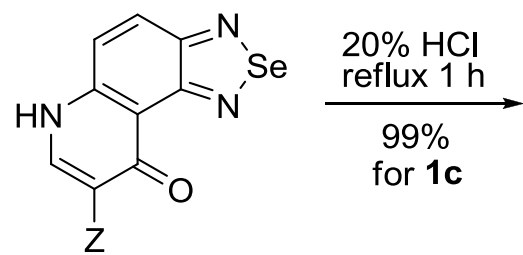

\begin{tabular}{l|ll}
$\mathbf{1}$ & \multicolumn{1}{|c}{ Z } & Yield (\%) \\
\hline $\mathbf{a}$ & $\mathrm{H}$ & 81 \\
$\mathbf{b}$ & COOMe & 85 \\
$\mathbf{c}$ & COOEt & 88 \\
e & COMe & 84 \\
$\mathbf{f}$ & $\mathrm{CN}$ & 66
\end{tabular}<smiles>O=C(O)c1c[nH]c2ccc3n[se]nc3c2c1=O</smiles>

7

\section{Scheme 3}

No linear annulation was observed during the thermal cyclization. The angular annulation of the selenadiazole and pyridone ring was confirmed by the coupling constants ${ }^{3} J_{\mathrm{HH}}$ (about $9.5 \mathrm{~Hz}$ ) resulting from ortho interaction of the benzene ring protons. Temperature of the cyclization (260 ${ }^{\circ} \mathrm{C}$ ), reaction time, purity of starting enamines and their appropriate dilution in suitable solvent $\left(\mathrm{Ph}_{2} \mathrm{O}\right)$ were necessary for the successful ring closure. The best dilution of enamines 6a-c,e in diphenylether was found to be 1:20 ( $1 \mathrm{~g}$ of starting enamine to $20 \mathrm{~mL} \mathrm{of} \mathrm{Ph}_{2} \mathrm{O}$ ). In this case, cyclization was complete within relatively short reaction times (20-60 min.). However, derivative $6 \mathbf{f}$ required dilution of 1:100 due to a longer reaction time $(4 \mathrm{~h})$ in order to avoid probable carbonization and degradation.

$[1,2,5]$ Selenadiazolo[3,4-f]quinoline-8-carboxylic acid 7 was accessed by acid hydrolysis of the corresponding ethyl ester 1c in almost quantitative yield (Scheme 3). 


\section{Conclusions}

In summary, we have described an efficient synthesis of angularly annulated selenadiazolo[3,4$f$ ]quinolones by the application of the Gould-Jacobs reaction to 5-amino-2,1,3benzoselenadiazole. In the course of thermal cyclization no linear products were observed.

\section{Experimental Section}

General. Melting points were measured on Koffler block and are uncorrected. ${ }^{1} \mathrm{H}$ NMR and ${ }^{13} \mathrm{C}$ NMR spectra were recorded on Varian Mercury $300-\mathrm{MHz}$ spectrometer at $25{ }^{\circ} \mathrm{C}$ with $\mathrm{CDCl}_{3}$, DMSO- $d_{6}$ or TFA- $d$ as solvents at operation frequency $300 \mathrm{MHz}$ for ${ }^{1} \mathrm{H}$ and $75.5 \mathrm{MHz}$ for ${ }^{13} \mathrm{C}$ nuclei. Chemical shifts are reported in parts per million ( $\mathrm{ppm}$ ) relative to internal standard TMS. Coupling constants $(J)$ are given in Hz. Infrared spectra were recorded on a Nicolet model NEXUS 470 FT-IR spectrometer in $\mathrm{KBr}$ pellets with absorption in $\mathrm{cm}^{-1}$. Elemental analyses were determined with a Thermo Fingion $\mathrm{CHNS}(\mathrm{O}) 1112$ instrument.

The alkoxymethylene derivatives $\mathbf{5 b}, \mathbf{c}, \mathbf{d}, \mathbf{f}$ are commercially available, while compounds $\mathbf{5 a}, \mathbf{e}, \mathbf{g - i}$ were synthetized by condensation of methyl or ethyl orthoformiate (according to the alkyl in the ester group from second reactant to avoid transesterification) with corresponding methylene compound. ${ }^{24,25}$

5-Amino-2,1,3-benzoselenadiazole (4). To a suspension of 2,4-nitroaniline (2) (22.0 g, 120.1 $\mathrm{mmol})$ in ethanol $(350 \mathrm{ml})$, freshly activated Raney nickel catalyst was added and the resulting suspension was heated to $50{ }^{\circ} \mathrm{C}$. Then hydrazine hydrate $(40 \mathrm{ml})$ was added dropwise at this temperature to ensure a gentle foaming of the reaction mixture. After the addition was complete, the reaction mixture was refluxed for $30 \mathrm{~min}$. to decompose the excess of hydrazine hydrate. After completion of the reaction (TLC, eluent $\mathrm{CHCl}_{3}: \mathrm{MeOH}=100: 1$ ), reaction mixture was cooled in an ice bath and filtered into ice cold concentrated $\mathrm{HCl}(60 \mathrm{ml})$. Formed precipitate was separated by suction, washed with ethanol and dried to afford dihydrochloride $\mathbf{3}$ as a slightly violet solid. Hydrochloride 3 ( $21.8 \mathrm{~g}, 111.1 \mathrm{mmol}$ ) was dissolved in water $(400 \mathrm{ml})$, charcoaled and filtered into a beaker. To the filtrate, solution of $\mathrm{SeO}_{2}(12.3 \mathrm{~g}, 111.1 \mathrm{mmol})$ in water $(30 \mathrm{ml})$ was added dropwise at room temperature. The reaction mixture was stirred for 10 min. and formed dark red suspension was subsequently alkalized with $20 \% \mathrm{NaOH}$ solution while cooling in an ice bath. The resulting precipitate was collected by suction, washed with water and dried to yield 5-amino-2,1,3-benzoselenadiazole (4) as orange solid (20.0 g, 91\%), mp 147-148 ${ }^{\circ} \mathrm{C}$ (lit., $\left.{ }^{13,14} 149-150{ }^{\circ} \mathrm{C}\right) .{ }^{1} \mathrm{H}$ NMR $\left(300 \mathrm{MHz}\right.$, DMSO- $\left.d_{6}\right): \delta_{\mathrm{H}} 6.59\left(1 \mathrm{H}, \mathrm{d},{ }^{4} \mathrm{~J}=2.2 \mathrm{~Hz}, 4-\mathrm{H}\right), 7.16$ $\left(1 \mathrm{H}, \mathrm{dd},{ }^{3} J=9.5 \mathrm{~Hz},{ }^{4} J=2.2 \mathrm{~Hz}, 6-\mathrm{H}\right), 7.55\left(1 \mathrm{H}, \mathrm{d},{ }^{3} \mathrm{~J}=9.5 \mathrm{~Hz}, 7-\mathrm{H}\right) ;{ }^{13} \mathrm{C}$ NMR $(300 \mathrm{MHz}$, DMSO- $\left.d_{6}\right): \delta_{\mathrm{C}} 97.0,123.8,126.5,149.4,155.8,159.3$. 
(Benzoselenadiazol-5-ylamino)ethene derivatives (6a-i). General procedure

A mixture of 5-amino-2,1,3-benzoselenadiazole (4) (1.98 g, $10 \mathrm{mmol})$ and the corresponding activated enolether 5a-i $(11 \mathrm{mmol})$ was refluxed in methanol or ethanol $(35 \mathrm{ml})$ until the amine 4 was consumed (TLC, eluent $\mathrm{CHCl3}: \mathrm{MeOH}=100: 1$ ). Products were precipitated during reflux and after cooling to room temperature the precipitate was collected by suction, washed with methanol or ethanol and dried. Recrystallization from hot dimethylsulphoxide without addition of charcoal and filtration yielded products $\mathbf{6 a - i}$.

5-[(2,1,3-Benzoselenadiazol-5-ylamino)methylene]-2,2-dimethyl-1,3-dioxane-4,6-dione (6a). Yellow-orange plates, yield $78 \%, 2.74 \mathrm{~g}, \mathrm{mp} 225-227{ }^{\circ} \mathrm{C}$; IR $\left(v_{\max }, \mathrm{cm}^{-1}\right)$ : 1728, 1674, 1621, 1517, 1429, 1266. ${ }^{1} \mathrm{H}$ NMR $\left(300 \mathrm{MHz}, \mathrm{CDCl}_{3}\right): \delta_{\mathrm{H}} 1.78\left(6 \mathrm{H}, \mathrm{s}, 2 \times \mathrm{CH}_{3}\right), 7.52\left(1 \mathrm{H}, \mathrm{dd},{ }^{3} J=9.5\right.$ $\left.\mathrm{Hz},{ }^{4} J=1.5 \mathrm{~Hz}, 6-\mathrm{H}\right), 7.73(1 \mathrm{H}, \mathrm{s}, 4-\mathrm{H}), 7.94\left(7-\mathrm{H}, \mathrm{d},{ }^{3} J=9.4 \mathrm{~Hz}, 1 \mathrm{H}\right), 8.78\left(1 \mathrm{H}, \mathrm{d},{ }^{3} J=14.0\right.$ $\mathrm{Hz},=\mathrm{CH}-), 11.40\left(1 \mathrm{H}\right.$, br d, NH). ${ }^{13} \mathrm{C} \mathrm{NMR}\left(75 \mathrm{MHz}, \mathrm{CDCl}_{3}\right): \delta_{\mathrm{C}} 27.1,89.1,105.4,108.8$, 122.6, 125.0, 138.9, 152.2, 163.0, 165.2. Anal. Calcd. for $\mathrm{C}_{13} \mathrm{H}_{11} \mathrm{~N}_{3} \mathrm{O}_{4} \mathrm{Se}$ (352.21): C, 44.33; $\mathrm{H}$, 3.15 ; N, 11.93\%. Found: C, 44.45; H, 3.17; N, 11.98\%.

Dimethyl 2-[(2,1,3-benzoselenadiazol-5-ylamino)methylene]propanedioate (6b). Yellow solid, yield $72 \%, 2.44 \mathrm{~g}$, mp 197-199 ${ }^{\circ} \mathrm{C}$; IR $\left(v_{\max }, \mathrm{cm}^{-1}\right): 1689,1650,1647,1612,1604 .{ }^{1} \mathrm{H}$ NMR $\left(300 \mathrm{MHz}, \mathrm{CDCl}_{3}\right): \delta_{\mathrm{H}} 3.82\left(3 \mathrm{H}, \mathrm{s}, \mathrm{OCH}_{3}\right), 3.89\left(3 \mathrm{H}, \mathrm{s}, \mathrm{OCH}_{3}\right), 7.35\left(1 \mathrm{H}, \mathrm{dd},{ }^{3} J=9.5 \mathrm{~Hz}\right.$, $\left.{ }^{4} J=2.3 \mathrm{~Hz}, 6-\mathrm{H}\right), 7.53\left(1 \mathrm{H}, \mathrm{d},{ }^{4} J=2.0 \mathrm{~Hz}, 4-\mathrm{H}\right), 7.83\left(1 \mathrm{H}, \mathrm{d},{ }^{3} J=9.5 \mathrm{~Hz}, 7-\mathrm{H}\right), 8.62\left(1 \mathrm{H}, \mathrm{d},{ }^{3} J=\right.$ $13.3 \mathrm{~Hz},=\mathrm{CH}-), 11.14(1 \mathrm{H}, \mathrm{br} \mathrm{d}, \mathrm{NH}) .{ }^{13} \mathrm{C} \mathrm{NMR}\left(75 \mathrm{MHz}, \mathrm{CDCl}_{3}\right): \delta_{\mathrm{C}} 51.7,51.9,95.7,105.8$, 123.6, 124.6, 140.2, 150.8, 158.3, 160.3, 165.3,169.1. Anal. Calcd. for $\mathrm{C}_{12} \mathrm{H}_{11} \mathrm{~N}_{3} \mathrm{O}_{4} \mathrm{Se}(340.20)$ : C, 42.37; H, 3.26; N, 12.35\%. Found: C, 42.26; H, 3.29; N, $12.31 \%$.

Diethyl 2-[(2,1,3-benzoselenadiazol-5-ylamino)methylene]propanedioate (6c). Yellow needles, yield 69\%, $2.54 \mathrm{~g}, \mathrm{mp} 167-169{ }^{\circ} \mathrm{C}$; IR $\left(v_{\max }, \mathrm{cm}^{-1}\right): 1686,1643,1617,1603,1422 .{ }^{1} \mathrm{H}$ NMR $\left(300 \mathrm{MHz}, \mathrm{CDCl}_{3}\right): \delta_{\mathrm{H}} 1.36\left(3 \mathrm{H}, \mathrm{t},{ }^{3} J=7.1 \mathrm{~Hz}, \mathrm{OCH}_{2} \mathrm{CH}_{3}\right), 1.40\left(3 \mathrm{H}, \mathrm{t},{ }^{3} J=7.1 \mathrm{~Hz}\right.$, $\left.\mathrm{OCH}_{2} \mathrm{CH}_{3}\right), 4.28\left(2 \mathrm{H}, \mathrm{q},{ }^{3} \mathrm{~J}=7.1 \mathrm{~Hz}, \mathrm{OCH}_{2} \mathrm{CH}_{3}\right), 4.35\left(2 \mathrm{H}, \mathrm{q},{ }^{3} \mathrm{~J}=7.1 \mathrm{~Hz}, \mathrm{OCH}_{2} \mathrm{CH}_{3}\right), 7.34(1 \mathrm{H}$, $\left.\mathrm{dd},{ }^{3} J=9.5 \mathrm{~Hz},{ }^{4} J=2.3 \mathrm{~Hz}, 6-\mathrm{H}\right), 7.51\left(4-\mathrm{H}, \mathrm{d},{ }^{4} J=2.0 \mathrm{~Hz}, 1 \mathrm{H}\right), 7.84\left(7-\mathrm{H}, \mathrm{d},{ }^{3} J=9.5 \mathrm{~Hz}, 1 \mathrm{H}\right)$, $8.60\left(1 \mathrm{H}, \mathrm{d},{ }^{3} \mathrm{~J}=13.2 \mathrm{~Hz},=\mathrm{CH}-\right), 11.12\left(1 \mathrm{H}\right.$, br d, NH). ${ }^{13} \mathrm{C}$ NMR $\left(75 \mathrm{MHz}, \mathrm{CDCl}_{3}\right): \delta_{\mathrm{C}} 14.2$, $14.4,60.4,60.8,96.5,105.4,123.8,124.5,140.4,150.3,158.3,160.4$, 165.0, 168.8. Anal. Calcd. for $\mathrm{C}_{14} \mathrm{H}_{15} \mathrm{~N}_{3} \mathrm{O}_{4} \mathrm{Se}(368.25)$ : C, 45.66; H, 4.11; N, 11.40\%. Found: C, 45.62; H, 4.19; N, $11.48 \%$. 3-[(2,1,3-Benzoselenadiazol-5-ylamino)methylene] pentane-2,4-dione (6d). Yellow solid, yield 70\%, $2.15 \mathrm{~g}, \mathrm{mp} 218-220{ }^{\circ} \mathrm{C}$; IR $\left(v_{\max }, \mathrm{cm}^{-1}\right): 1629,1612,1512,1397,1319 .{ }^{1} \mathrm{H}$ NMR $(300$ $\left.\mathrm{MHz}, \mathrm{CDCl}_{3}\right): \delta_{\mathrm{H}} 2.44\left(3 \mathrm{H}, \mathrm{s}, \mathrm{COCH}_{3}\right), 2.59\left(3 \mathrm{H}, \mathrm{s}, \mathrm{COCH}_{3}\right), 7.36\left(1 \mathrm{H}, \mathrm{dd},{ }^{3} J=9.5 \mathrm{~Hz},{ }^{4} J=2.3\right.$ $\mathrm{Hz}, 6-\mathrm{H}), 7.57\left(1 \mathrm{H}, \mathrm{d},{ }^{4} J=2.2 \mathrm{~Hz}, 4-\mathrm{H}\right), 7.88\left(1 \mathrm{H}, \mathrm{d},{ }^{3} J=9.5 \mathrm{~Hz}, 7-\mathrm{H}\right), 8.32\left(1 \mathrm{H}, \mathrm{d},{ }^{3} J=12.3\right.$ $\mathrm{Hz},=\mathrm{CH}-), 12.77(1 \mathrm{H}$, br d, $\mathrm{NH}) .{ }^{13} \mathrm{C} \mathrm{NMR}\left(75 \mathrm{MHz}, \mathrm{CDCl}_{3}\right): \delta_{\mathrm{C}} 27.4,32.1,106.4,114.8$, 124.0, 124.8, 140.4, 150.2, 158.4, 160.2, 194.9, 201.8. Anal. Calcd. for $\mathrm{C}_{12} \mathrm{H}_{11} \mathrm{~N}_{3} \mathrm{O}_{2} \mathrm{Se}$ (308.20): C, 46.77; H, 3.60; N, 13.60\%. Found: C, 46.71; H, 3.62; N, 13.68\%.

(E/Z)-Ethyl 2-[(2,1,3-benzoselenadiazol-5-ylamino)methylene]-3-oxobutanoate (6e). Yellow solid, yield 70\%, $2.36 \mathrm{~g}, \mathrm{mp} 163-165{ }^{\circ} \mathrm{C}, E: Z=10: 1$; IR $\left(v_{\max }, \mathrm{cm}^{-1}\right): 1708,1635,1616,1588$, 1511. ${ }^{1} \mathrm{H}$ NMR $\left(300 \mathrm{MHz}, \mathrm{CDCl}_{3}\right) E$-isomer: $\delta_{\mathrm{H}} 1.38\left(3 \mathrm{H}, \mathrm{t},{ }^{3} J=7.1 \mathrm{~Hz}, \mathrm{OCH}_{2} \mathrm{CH}_{3}\right), 2.59(3 \mathrm{H}$, $\left.\mathrm{s}, \mathrm{COCH}_{3}\right), 4.30\left(2 \mathrm{H}, \mathrm{q},{ }^{3} J=7.1 \mathrm{~Hz}, \mathrm{OCH}_{2} \mathrm{CH}_{3}\right), 7.38\left(1 \mathrm{H}, \mathrm{dd},{ }^{3} J=9.5 \mathrm{~Hz},{ }^{4} J=2.3 \mathrm{~Hz}, 6-\mathrm{H}\right)$, 
$7.58\left(1 \mathrm{H}, \mathrm{d},{ }^{4} J=2.0 \mathrm{~Hz}, 4-\mathrm{H}\right), 7.85\left(1 \mathrm{H}, \mathrm{d},{ }^{3} J=9.5 \mathrm{~Hz}, 7-\mathrm{H}\right), 8.59\left(1 \mathrm{H}, \mathrm{d},{ }^{3} J=12.7 \mathrm{~Hz},=\mathrm{CH}-\right)$, $12.80(1 \mathrm{H}$, br d, $\mathrm{NH}) .{ }^{13} \mathrm{C} \mathrm{NMR}\left(75 \mathrm{MHz}, \mathrm{CDCl}_{3}\right) E$-isomer: $\delta_{\mathrm{C}} 14.5,31.3,60.3,104.6,106.6$, 123.8, 124.6, 140.3, 150.6, 158.4, 160.3, 166.4, 200.9. Anal. Calcd. for $\mathrm{C}_{13} \mathrm{H}_{13} \mathrm{~N}_{3} \mathrm{O}_{3} \mathrm{Se}(338.23)$ : C, 46.16; H, 3.87; N, 12.42\%. Found: C, 46.10; H, 3.88; N, $12.21 \%$.

(E/Z)-Ethyl 3-(2,1,3-benzoselenadiazol-5-ylamino)-2-cyanoprop-2-enoate (6f). Yellow solid, yield 64\%, $2.05 \mathrm{~g}$, mp 250-255 ${ }^{\circ} \mathrm{C}, E: Z=4: 1$, IR $\left(v_{\max }, \mathrm{cm}^{-1}\right): 2213,1673,1635,1418,1382 .{ }^{1} \mathrm{H}$ NMR (300 MHz, DMSO- $\left.d_{6}\right) E$-isomer: $\delta_{\mathrm{H}} 1.27\left(3 \mathrm{H}, \mathrm{t},{ }^{3} J=7.3 \mathrm{~Hz}, \mathrm{OCH}_{2} \mathrm{CH}_{3}\right), 4.22\left(2 \mathrm{H}, \mathrm{q},{ }^{3} J=\right.$ $\left.7.3 \mathrm{~Hz}, \mathrm{OCH}_{2} \mathrm{CH}_{3}\right), 7.73-7.90(3 \mathrm{H}, \mathrm{m}, \mathrm{ArH}), 8.47\left(1 \mathrm{H}, \mathrm{d},{ }^{3} \mathrm{~J}=13.9 \mathrm{~Hz},=\mathrm{CH}-\right), 10.86(1 \mathrm{H}, \mathrm{br}$, $\mathrm{NH}) .{ }^{13} \mathrm{C}$ NMR $\left(75 \mathrm{MHz}, \mathrm{DMSO}-d_{6}\right) E$-isomer: $\delta_{\mathrm{C}} 14.1,60.4,77.1,107.1,115.3,123.5,123.7$, 140.7, 151.9, 157.6, 159.5, 163.9. Anal. Calcd. for $\mathrm{C}_{12} \mathrm{H}_{10} \mathrm{~N}_{4} \mathrm{O}_{2} \mathrm{Se}$ (321.20): C, 44.87; H, 3.14; N, $17.44 \%$. Found: C, 44.83; H, 3.12; N, $17.41 \%$.

(E/Z)-Methyl 2-[(2,1,3-benzoselenadiazol-5-ylamino)methylene]-3-oxobutanoate (6g). Yellow solid, yield $68 \%, 2.20 \mathrm{~g}, \mathrm{mp} 168-171{ }^{\circ} \mathrm{C}, E: Z=12: 1, \mathrm{IR}\left(v_{\max }, \mathrm{cm}^{-1}\right): 1712,1638,1619$, 1585. ${ }^{1} \mathrm{H}$ NMR $\left(300 \mathrm{MHz}, \mathrm{CDCl}_{3}\right) E$-isomer: $\delta_{\mathrm{H}} 2.59\left(3 \mathrm{H}, \mathrm{s}, \mathrm{COCH}_{3}\right), 3.83\left(3 \mathrm{H}, \mathrm{s}, \mathrm{OCH}_{3}\right), 7.38$ $\left(1 \mathrm{H}, \mathrm{dd},{ }^{3} J=9.5 \mathrm{~Hz},{ }^{4} J=2.3 \mathrm{~Hz}, 6-\mathrm{H}\right), 7.59\left(1 \mathrm{H}, \mathrm{d},{ }^{4} J=2.2 \mathrm{~Hz}, 4-\mathrm{H}\right), 7.85\left(1 \mathrm{H}, \mathrm{d},{ }^{3} J=9.5 \mathrm{~Hz}\right.$, 7-H), $8.59\left(1 \mathrm{H}, \mathrm{d},{ }^{3} \mathrm{~J}=12.7 \mathrm{~Hz},=\mathrm{CH}-\right), 12.81\left(1 \mathrm{H}\right.$, br d, NH). ${ }^{13} \mathrm{C}$ NMR $\left(75 \mathrm{MHz}, \mathrm{CDCl}_{3}\right) E-$ isomer: $\delta_{\mathrm{C}} 31.3,51.5,104.3,106.8,123.7,124.6,140.3,150.8,158.4,160.3,166.7,200.9$. Anal. Calcd. for $\mathrm{C}_{12} \mathrm{H}_{11} \mathrm{~N}_{3} \mathrm{O}_{3} \mathrm{Se}$ (324.20): C, 44.46; H, 3.42; N, 12.96\%. Found: C, 44.50; H, 3.40; N, $12.87 \%$.

(E/Z)-Methyl 3-(2,1,3-benzoselenadiazol-5-ylamino)-2-cyanoprop-2-enoate (6h). Yellow solid, yield 56\%, $1.72 \mathrm{~g}, \mathrm{mp} 284-286{ }^{\circ} \mathrm{C}, E: Z=3: 1$, IR $\left(v_{\max }, \mathrm{cm}^{-1}\right): 2214,1715,1631,1529 .{ }^{1} \mathrm{H}$ NMR $\left(300 \mathrm{MHz}, \mathrm{DMSO}-d_{6}\right) E$-isomer: $\delta_{\mathrm{H}} 3.75\left(3 \mathrm{H}, \mathrm{s}, \mathrm{OCH}_{3}\right), 7.73-7.93(3 \mathrm{H}, \mathrm{m}, \mathrm{ArH}), 8.50$ $\left(1 \mathrm{H}, \mathrm{d},{ }^{3} \mathrm{~J}=13.8 \mathrm{~Hz},=\mathrm{CH}-\right), 10.89(1 \mathrm{H}, \mathrm{br} \mathrm{d}, \mathrm{NH}) .{ }^{13} \mathrm{C}$ NMR $\left(75 \mathrm{MHz}, \mathrm{DMSO}-d_{6}\right) E$-isomer: $\delta_{\mathrm{C}}$ 51.7, 76.7, 107.3, 115.3, 123.5, 123.7, 140.7, 152.1, 157.6, 159.5, 164.4. Anal. Calcd. for $\mathrm{C}_{11} \mathrm{H}_{8} \mathrm{~N}_{4} \mathrm{O}_{2} \mathrm{Se}$ (307.17): C, 43.01; H, 2.63; N, 18.24\%. Found: C, 42.92; H, 2.58; N, $18.25 \%$.

(E/Z)-2-[(2,1,3-Benzoselenadiazol-5-ylamino)methylene]-3-oxobutanenitrile (6i). Yellow solid, yield 76\%, $2.21 \mathrm{~g}, \mathrm{mp} 273-278{ }^{\circ} \mathrm{C}, E: Z=1: 2$, IR $\left(v_{\max }, \mathrm{cm}^{-1}\right): 2204,1679,1651,1622$, 1604. ${ }^{1} \mathrm{H}$ NMR (300 MHz, DMSO- $\left.d_{6}\right) Z$-isomer: $\delta_{\mathrm{H}} 2.36\left(3 \mathrm{H}, \mathrm{s}, \mathrm{CH}_{3}\right), 7.76-7.96(3 \mathrm{H}, \mathrm{m}, \mathrm{ArH})$, $8.52\left(1 \mathrm{H}, \mathrm{d},{ }^{3} J=13.6 \mathrm{~Hz},=\mathrm{CH}-\right), 10.84(1 \mathrm{H}, \mathrm{br} \mathrm{d}, \mathrm{NH}) .{ }^{13} \mathrm{C}$ NMR $\left(300 \mathrm{MHz}, \mathrm{DMSO}-d_{6}\right) Z-$ isomer: $\delta_{\mathrm{C}} 26.0,88.6,107.3,115.8,123.4,123.8,140.7,151.3,159.5,163.9,191.1$. Anal. Calcd. for $\mathrm{C}_{11} \mathrm{H}_{8} \mathrm{~N}_{4} \mathrm{OSe}$ (291.17): C, 45.38; H, 2.77; N, 19.24\%. Found: C, 45.39; H, 2.79; N, 19.30\%.

\section{$[1,2,5]$ selenadiazolo[3,4-f]quinoline-9(6H)-ones (1a-c, e, f). General procedure}

A mixture of (benzoselenadiazol-5-ylamino)ethene derivative 6a-c, e, f (1.0 g) and diphenyl ether $(20 \mathrm{ml}, 100 \mathrm{ml}$ for $6 \mathbf{f})$ was heated at $260^{\circ} \mathrm{C}$ in an oil bath. After cooling the reaction mixture, the precipitated solid was collected by suction, washed with dichloromethane several times and dried in a vacuum oven at $80^{\circ} \mathrm{C}$ for 6 hours to remove diphenyl ether.

[1,2,5]Selenadiazolo[3,4-f]quinoline-9(6H)-one (1a). Brownish solid, yield 81\%, $0.57 \mathrm{~g} \mathrm{mp}$ 243-245 ${ }^{\circ} \mathrm{C}$, IR $\left(v_{\max }, \mathrm{cm}^{-1}\right): 3046,1621,1557,1462,836 .{ }^{1} \mathrm{H}$ NMR $(300 \mathrm{MHz}, \mathrm{TFA}-d): \delta_{\mathrm{H}} 7.31$ $\left(1 \mathrm{H}, \mathrm{d},{ }^{3} J=6.9 \mathrm{~Hz}, 8-\mathrm{H}\right), 7.86\left(1 \mathrm{H}, \mathrm{d},{ }^{3} J=9.8 \mathrm{~Hz}, 4-\mathrm{H}\right), 8.07\left(1 \mathrm{H}, \mathrm{d},{ }^{3} J=9.8 \mathrm{~Hz}, 5-\mathrm{H}\right), 8.43$ 
$\left(1 \mathrm{H}, \mathrm{d},{ }^{3} J=6.9 \mathrm{~Hz}, 7-\mathrm{H}\right) .{ }^{13} \mathrm{C}$ NMR $(300 \mathrm{MHz}, \mathrm{TFA}-d): \delta_{\mathrm{C}} 114.5,115.1,125.9,133.2,146.0$, 146.2, 157.5, 159.0, 173.6. Anal. Calcd. for $\mathrm{C}_{9} \mathrm{H}_{5} \mathrm{~N}_{3} \mathrm{OSe}$ (250.12): C, 43.22; H, 2.01; N, 16.80\%. Found: C, 43.15; H, 1.97; N, 16.85\%.

Methyl 6,9-dihydro-9-oxo-[1,2,5] selenadiazolo[3,4-f]quinoline-8-carboxylate (1b). Yellowbrown solid, yield $85 \%, 0.77 \mathrm{~g}, \mathrm{mp} 340-348^{\circ} \mathrm{C}$, IR $\left(v_{\max }, \mathrm{cm}^{-1}\right): 3046,1735,1686,1570 .{ }^{1} \mathrm{H}$ NMR $(300 \mathrm{MHz}, \mathrm{TFA}-d): \delta_{\mathrm{H}} 3.84\left(3 \mathrm{H}, \mathrm{s}, \mathrm{OCH}_{3}\right), 7.87\left(1 \mathrm{H}, \mathrm{d},{ }^{3} J=9.7 \mathrm{~Hz}, 4-\mathrm{H}\right), 8.19\left(1 \mathrm{H}, \mathrm{d},{ }^{3} J\right.$ $=9.6 \mathrm{~Hz}, 5-\mathrm{H}), 9.08(1 \mathrm{H}, \mathrm{s}, 7-\mathrm{H}) .{ }^{13} \mathrm{C}$ NMR $(300 \mathrm{MHz}, \mathrm{TFA}-d): \delta_{\mathrm{C}} 56.4,113.0,115.9,125.3$, 135.4, 147.7, 147.9, 154.5, 160.1, 169.0, 173.9. Anal. Calcd. for $\mathrm{C}_{11} \mathrm{H}_{7} \mathrm{~N}_{3} \mathrm{O}_{3} \mathrm{Se}$ (308.16): C, 42.87; H, 2.29; N, 13.64\%. Found: C, 42.81; H, 2.23; N, 13.67\%.

Ethyl 6,9-dihydro-9-oxo-[1,2,5]selenadiazolo[3,4-f]quinoline-8-carboxylate (1c). Yellow solid, yield $88 \%, 0.77 \mathrm{~g}$, mp 289-294 ${ }^{\circ} \mathrm{C}$, IR $\left(v_{\max }, \mathrm{cm}^{-1}\right): 3043,1715,1685,1630,1561 .{ }^{1} \mathrm{H}$ NMR $(300 \mathrm{MHz}, \mathrm{TFA}-d): \delta_{\mathrm{H}} 1.20\left(3 \mathrm{H}, \mathrm{t},{ }^{3} J=7.1 \mathrm{~Hz}, \mathrm{OCH}_{2} \mathrm{CH}_{3}\right), 4.37\left(2 \mathrm{H}, \mathrm{q},{ }^{3} J=7.1 \mathrm{~Hz}\right.$, $\left.\mathrm{OCH}_{2} \mathrm{CH}_{3}\right), 7.93\left(1 \mathrm{H}, \mathrm{d},{ }^{3} \mathrm{~J}=9.7 \mathrm{~Hz}, 4-\mathrm{H}\right), 8.23\left(1 \mathrm{H}, \mathrm{d},{ }^{3} \mathrm{~J}=9.7 \mathrm{~Hz}, 5-\mathrm{H}\right), 9.13(1 \mathrm{H}, \mathrm{s}, 7-\mathrm{H}) .{ }^{13} \mathrm{C}$ NMR (300 MHz, TFA-d): $\delta_{\mathrm{C}} 14.6,67.8,113.2,116.0,125.4,135.4,147.6,147.9,154.4,160.2$, 168.8, 174.1. Anal. Calcd. for $\mathrm{C}_{12} \mathrm{H}_{9} \mathrm{~N}_{3} \mathrm{O}_{3} \mathrm{Se}$ (322.18): C, 44.74; H, 2.82; N, 13.04\%. Found: C, 44.70; H, 2.89; N, 13.10\%.

8-Acetyl-[1,2,5]selenadiazolo[3,4-f]quinoline-9(6H)-one (1e). Yellow-brown solid, yield 84\%, $0.72 \mathrm{~g}, \mathrm{mp}>350{ }^{\circ} \mathrm{C}$, IR $\left(v_{\max }, \mathrm{cm}^{-1}\right): 3044,1666,1647,1560 .{ }^{1} \mathrm{H}$ NMR $(300 \mathrm{MHz}, \mathrm{TFA}-d): \delta_{\mathrm{H}}$ $2.44\left(3 \mathrm{H}, \mathrm{s}, \mathrm{COCH}_{3}\right), 7.70\left(1 \mathrm{H}, \mathrm{d},{ }^{3} J=9.7 \mathrm{~Hz}, 4-\mathrm{H}\right), 8.00\left(1 \mathrm{H}, \mathrm{d},{ }^{3} J=9.7 \mathrm{~Hz}, 5-\mathrm{H}\right), 8.94(1 \mathrm{H}, \mathrm{s}$, $7-\mathrm{H}) .{ }^{13} \mathrm{C}$ NMR $(300 \mathrm{MHz}, \mathrm{TFA}-d): \delta_{\mathrm{C}} 29.5,115.2,119.5,124.6,134.7,146.7,148.0,155.0$, 159.1, 173.2, 202.9. Anal. Calcd. for $\mathrm{C}_{11} \mathrm{H}_{7} \mathrm{~N}_{3} \mathrm{O}_{2} \mathrm{Se}$ (292.16): C, 45.22; H, 2.42; N, 14.48\%. Found: C, 45.30; H, 2.50; N, 14.31\%.

6,9-Dihydro-6-oxo-[1,2,5] selenadiazolo[3,4-f]quinoline-8-carbonitrile (1f). Brown solid, yield $66 \%, 0.56 \mathrm{~g}, \mathrm{mp}>350{ }^{\circ} \mathrm{C}$; IR $\left(v_{\max }, \mathrm{cm}^{-1}\right): 3046,2224,1628,1566 .{ }^{1} \mathrm{H}$ NMR $(300 \mathrm{MHz}$, TFAd): $\delta_{\mathrm{H}} 8.03\left(1 \mathrm{H}, \mathrm{d},{ }^{3} J=9.7 \mathrm{~Hz}, 4-\mathrm{H}\right), 8.27\left(1 \mathrm{H}, \mathrm{d},{ }^{3} J=9.7 \mathrm{~Hz}, 5-\mathrm{H}\right), 9.02(1 \mathrm{H}, \mathrm{s}, 7-\mathrm{H}) .{ }^{13} \mathrm{C}$ NMR $(300 \mathrm{MHz}, \mathrm{TFA}-d): \delta_{\mathrm{C}} 98.3,108.8,111.7,122.8,133.3,144.8,147.9,153.7,156.5,171.7$. Anal. Calcd. for $\mathrm{C}_{10} \mathrm{H}_{4} \mathrm{~N}_{4} \mathrm{OSe}$ (275.13): C, 43.66; H, 1.57; N, 20.36\%. Found: C, 43.59; H, 1.45; N, $20.40 \%$.

\section{6,9-Dihydro-9-oxo-[1,2,5]selenadiazolo[3,4-f]quinoline-8-carboxylic acid (7)}

A mixture of ethyl ester $1 \mathrm{c}(1.0 \mathrm{~g}, 3.1 \mathrm{mmol})$ and $20 \%$ hydrochloric acid $(50 \mathrm{ml})$ was refluxed for 1 hour. After cooling the reaction mixture, water $(30 \mathrm{ml})$ was added and the precipitate was collected by suction, washed with water and dried to yield yellowish solid $(99 \%, 0.90 \mathrm{~g}), \mathrm{mp}>$ $335{ }^{\circ} \mathrm{C}$ (sublimation), IR ( $\left.v_{\max }, \mathrm{cm}^{-1}\right): 3406,1695,1690,1574 .{ }^{1} \mathrm{H}$ NMR $(300 \mathrm{MHz}, \mathrm{TFA}-d): \delta_{\mathrm{H}}$ $8.10\left(1 \mathrm{H}, \mathrm{d},{ }^{3} J=9.7 \mathrm{~Hz}, 4-\mathrm{H}\right), 8.42\left(1 \mathrm{H}, \mathrm{d},{ }^{3} J=9.7 \mathrm{~Hz}, 5-\mathrm{H}\right), 9.35(1 \mathrm{H}, \mathrm{s}, 7-\mathrm{H}) .{ }^{13} \mathrm{C}$ NMR $(300$ MHz, TFA-d): $\delta_{\mathrm{C}} 112.9,116.0,125.4,135.7,148.2,148.7,154.7,160.1,170.8$, 174.6. Anal. Calcd. for $\mathrm{C}_{10} \mathrm{H}_{5} \mathrm{~N}_{3} \mathrm{O}_{3} \mathrm{Se}$ (294.13): C, 40.84; H, 1.71; N, 14.39\%. Found: C, 40.79; H, 1.75; N, $14.33 \%$. 


\section{Acknowledgements}

The authors are grateful to the Slovak Grant Agency for Science (contract No. 1/0660/11) and to Slovak Research and Development Agency (contract No. APVV-0339-10) for financial support and to Assoc. Prof. Dr. M. Breza for quantum calculations.

\section{References}

1. Milata, V.; Claramunt, R. M.; Elguero, J.; Zálupský, P. Targets Heterocycl. Syst. 2000, 4, 167.

2. Boteva, A.; Krasnykh, O. Chem. Heterocycl. Compd. 2009, 45, 757.

3. Barbieriková, Z.; Bella, M.; Kučerák, J.; Milata, V.; Jantová, S.; Dvoranová, D.; Veselá, M; Staško, A.; Brezová, V. Photochem. Photobiol. 2011, 87, 32.

4. Jantová, S.; Koňariková, K.; Letašiová, S.; Paulovičová, E.; Milata, V.; Brezová, V. J. Photochem. Photobiol. B: Biol. 2011, 102, 77.

5. Staško, A.; Zalibera, M.; Barbieriková, Z.; Rimarčík, J.; Lukeš, V.; Bella, M.; Milata, V.; Brezová, V. Magn. Reson. Chem. 2011, 49, 168.

6. Barbieriková, Z.; Bella, M.; Lietava, J.; Dvoranová, D.; Staško, A.; Füzik, T.; Milata, V.; Jantová, S.; Brezová, V. J. Photochem. Photobiol. A: Chem. 2011, 224, 123.

7. Młochowski, J.; Kloc, K.; Lisiak, R.; Potaczek, P.; Wójtowicz, H. Arkivoc 2007, (vi), 14.

8. Mugesh, G.; du Mont, W.-W.; Sies, H. Chem. Rev. 2001, 101, 2125.

9. Nogueira, C. W.; Zeni, G.; Rocha, J. B. T. Chem. Rev. 2004, 104, 6255.

10. Grivas, S. Curr. Org. Chem. 2000, 4, 707.

11. Curran, T. T. in Name Reactions in Heterocyclic Chemistry; Li, J. J. Ed.; Wiley \& Sons: Hoboken, NJ, 2005; pp 423.

12. Saloň, J.; Milata, V.; Gatial, A.; Prónayová, N.; Leško, J.; Černuchová, P.; Rappoport, Z.; Vo-Thanh, G.; Loupy, A. Eur. J. Org. Chem. 2005, 4780.

13. Hinsberg, O.; Chem. Ber. 1889, 22, 2895.

14. Efros, L. S.; Todres-Selektor, Z. V. Zh. Obshch. Khim. 1957, 27, 983.

15. Gilman, H.; Broadbent, H. S. J. Am. Chem. Soc. 1948, 70, 2619.

16. Becke, A. D. J. Chem. Phys. 1993, 98, 5648.

17. Woon, D. E.; Dunning Jr. T. H. J. Chem. Phys. 1993, 98, 1358.

18. Wilson, A. K.; Woon, D. E.; Peterson, K. A.; Dunning, Jr. T. H. J. Chem. Phys. 1999, 110, 7667.

19. Frisch, M. J.; Trucks, G. W.; Schlegel, H. B.; Scuseria, G. E.; Robb, M. A.; Cheeseman, J. R. Gaussian 03 (Revision C1), Gaussian, Inc., Pittsburgh, PA, 2003.

20. Bella, M.; Schultz, M.; Milata, V.; Koňariková, K.; Breza, M. Tetrahedron 2010, 66, 8169.

21. Michalik, M. Z. Chem. 1985, 25, 287.

22. Couchouron, B.; Le Saint, J.; Courtot, P. Bull. Soc. Chim. Fr., Part 2, 1983, 3, 66. 
23. Leško, J.; Milata, V.; Schultz, M. Molecules 2000, 5, 937.

24. Saloň, J.; Milata, V.; Prónayová, N.; Leško, J. Monatsh. Chem. 2000, 131, 293.

25. Ohmori, J.; Sakamoto, S.; Kubota, H.; Shimizu-Sasamata, M.; Okada, M.; Kawasaki, S.; Hidaka, K.; Togami, J.; Furuya, T.; Murase, K. J. Med. Chem. 1994, 37, 467. 